\title{
A COMPREHENSIVE CHARACTERIZATION OF THE 70 VIRGINIS PLANETARY SYSTEM
}

\author{
Stephen R. Kane ${ }^{1}$, Tabetha S. Boyajian ${ }^{2}$, Gregory W. Henry ${ }^{3}$, Y. Katherina Feng ${ }^{4,5,6}$, Natalie R. Hinkel $^{1}$, \\ Debra A. Fischer ${ }^{2}$, Kaspar von Braun ${ }^{7}$, Andrew W. Howard ${ }^{8}$, and Jason T. Wright ${ }^{4,5}$ \\ ${ }^{1}$ Department of Physics \& Astronomy, San Francisco State University, 1600 Holloway Avenue, San Francisco, CA 94132, USA; skane@ sfsu.edu \\ ${ }^{2}$ Department of Astronomy, Yale University, New Haven, CT 06511, USA \\ ${ }^{3}$ Center of Excellence in Information Systems, Tennessee State University, 3500 John A. Merritt Blvd., Box 9501, Nashville, TN 37209, USA \\ ${ }_{5}^{4}$ Department of Astronomy and Astrophysics, Pennsylvania State University, 525 Davey Laboratory, University Park, PA 16802, USA \\ ${ }^{5}$ Center for Exoplanets \& Habitable Worlds, Pennsylvania State University, 525 Davey Laboratory, University Park, PA 16802, USA \\ ${ }^{6}$ Department of Astronomy \& Astrophysics, 1156 High Street, MS: UCO/LICK, University of California, Santa Cruz, CA 95064, USA \\ 7 Lowell Observatory, 1400 West Mars Hill Road, Flagstaff, Arizona 86001, USA \\ ${ }^{8}$ Institute for Astronomy, University of Hawaii, Honolulu, HI 96822, USA \\ Received 2015 January 6; accepted 2015 April 15; published 2015 June 8
}

\begin{abstract}
An on-going effort in the characterization of exoplanetary systems is the accurate determination of host star properties. This effort extends to the relatively bright host stars of planets discovered with the radial velocity method. The Transit Ephemeris Refinement and Monitoring Survey (TERMS) is aiding in these efforts as part of its observational campaign for exoplanet host stars. One of the first known systems is that of 70 Virginis, which harbors a jovian planet in an eccentric orbit. Here we present a complete characterization of this system with a compilation of TERMS photometry, spectroscopy, and interferometry. We provide fundamental properties of the host star through direct interferometric measurements of the radius (1.5\% uncertainty) and through spectroscopic analysis. We combined 59 new Keck HIRES radial velocity measurements with the 169 previously published from the ELODIE, Hamilton, and HIRES spectrographs, to calculate a refined orbital solution and construct a transit ephemeris for the planet. These newly determined system characteristics are used to describe the Habitable Zone of the system with a discussion of possible additional planets and related stability simulations. Finally, we present 19 years of precision robotic photometry that constrain stellar activity and rule out central planetary transits for a Jupiter-radius planet at the $5 \sigma$ level, with reduced significance down to an impact parameter of $b=0.95$.
\end{abstract}

Key words: planetary systems - stars: individual (70 Vir) - techniques: photometric - techniques: radial velocities

Supporting material: machine-readable table

\section{INTRODUCTION}

The exoplanet discoveries over the past couple of decades have revealed a particular need to understand the properties of the host stars. This is because the planetary parameters derived from the detection methods of radial velocities (RV) and transits rely heavily upon the mass and radius determinations of their parent stars. These are often determined using stellar models, but there are ongoing efforts to provide more direct measurements of the stellar properties through asteroseismology (Huber et al. 2014) and interferometry (Boyajian et al. 2012, 2013; von Braun et al. 2014). The importance of these measurements cannot be overstated since they not only affect the derived planetary properties but also the quantification of the Habitable Zone (HZ; Kasting et al. 1993; Kopparapu et al. 2013, 2014) and subsequent calculations of the fraction of stars with Earth-size planets in the HZ, or $\eta_{\oplus}$ (Dressing \& Charbonneau 2013; Kopparapu 2013; Petigura et al. 2013).

The Transit Ephemeris Refinement and Monitoring Survey (TERMS) is aiding in stellar characterization for the brightest stars as part of its program to improve exoplanetary orbital parameters (Kane \& von Braun 2008; Kane et al. 2009). Recent results include detailed spectroscopic and photometric analyses of the planet-hosting stars HD 38529 (Henry et al. 2013) and HD 192263 (Dragomir et al. 2012) and the identification of long-term activity cycles. TERMS observations have also led to the discovery of new planets in the HD 37605 (Wang et al. 2012) and HD 4203 (Kane et al. 2014) systems. These efforts are continuing with a focus on the brightest host stars, which tend to be those around which planets were discovered using the RV method.

One of the earliest exoplanet discoveries was that of the planet orbiting the bright $(V=5)$ star 70 Virginis (hereafter 70 Vir). The planet was discovered by Marcy \& Butler (1996) and lies in an eccentric $(e=0.4) 116$ day orbit. Perryman et al. (1996) subsequently used Hipparcos astrometry to constrain the inclination and thus determine that the companion is indeed sub-stellar in mass. Observations of $70 \mathrm{Vir}$ have continued since discovery, with RV data from Observatoire de HauteProvence published by Naef et al. (2004) and the complete Lick Observatory dataset compiled by Butler et al. (2006). A detailed characterization of the nearest and brightest exoplanet host stars is important because these continue to be those that are most suitable for potentially studying exoplanetary atmospheres for transiting planets.

Here we provide a detailed analysis of the 70 Vir system for both the star and the known planet. Section 2 describes new interferometric observations obtained using the Center for High Angular Resolution Astronomy (CHARA) Array. Section 3 combines these measurements with a new spectroscopic analysis of Keck/HIRES data to determine fundamental stellar properties of 70 Vir. Section 4 presents the addition of $\sim 60$ Keck/HIRES RV measurements to the existing time series, a revised Keplerian orbital solution, and the calculation of an accurate transit ephemeris. Section 5 uses the greatly improved system parameters to calculate the extent of the HZ and discusses the prospect of $\mathrm{HZ}$ planets in the system. Section 6 
Table 1

Log of Interferometric Observations For 70 Vir

\begin{tabular}{lccc}
\hline \hline UT Date & Baseline & \# of Obs & Calibrators \\
\hline 2013 Apr 03 & S1/E1 & 5 & HD 119288, HD 121560, \\
2013 Apr 04 & S1/E1 & 4 & HD 122386 \\
2014 Apr 20 & W1/E1 & 4 & HD 119550, HD 113022 \\
\hline
\end{tabular}

Note.Calibrator angular diameters from JSDC ${ }^{\mathrm{a}}$ are: $\theta_{\mathrm{HD} 113022}=0.39 \pm 0.03$, $\theta_{\mathrm{HD} 119288}=0.39 \pm 0.03, \theta_{\mathrm{HD} 119550}=0.38 \pm 0.03, \theta_{\mathrm{HD} 121560}=0.46 \pm 0.03$, and $\theta_{\mathrm{HD} 122386}=0.49 \pm 0.03$ mas. For details on the interferometric observations, see Section 2.

${ }^{a}$ Available at http://www.jmmc.fr/searchcal.

describes 19 years of precision robotic photometry that both rules out a planetary transit and shows that the long-term stellar activity is constant within 0.004 mag. We provide concluding remarks in Section 7.

\section{INTERFEROMETRIC OBSERVATIONS}

70 Vir (HD 117176; HR 5072; HIP 65721) is a bright $(V=4.97 ; H=3.24$; Johnson et al. 1968) and nearby (Hipparcos parallax of $55.60 \pm 0.24$ mas; van Leeuwen 2007) star. Our interferometric observations of $70 \mathrm{Vir}$ were conducted at the Georgia State University's Center for High Angular Resolution Astronomy (CHARA) Array and the Classic beam combiner in two-telescope mode operating in $H$-band (central wavelength $\lambda_{c}=1.67 \mu \mathrm{m}$; ten Brummelaar et al. 2005). We collected a total of 13 observations over the course of three nights: two nights in 2013 April using the S1E1 pair of telescopes, and one night in 2014 April using the W1E1 pair of telescopes. The S1E1 and E1W1 are the two longest telescope configurations available at CHARA, with baselines $B$ (distances between two telescopes) of $B_{\mathrm{S} 1 \mathrm{E} 1}=330 \mathrm{~m}$ and $B_{\mathrm{W} 1 \mathrm{E} 1}=313 \mathrm{~m}$.

Calibrator stars were observed in bracketed sequences with 70 Vir. We use the SearchCal software (Bonneau et al. 2006, 2011) to select calibrator stars based on their proximity in the sky with respect to the science target (within $\sim 8^{\circ}$ ). We observe a total of five calibrators with estimated angular sizes $\theta_{\text {est }}<0.5$ mas in order to minimize systematic errors which could be introduced by the calibrator's estimated sizes (van Belle \& van Belle 2005). A log of the observations along with calibrator information can be found in Table 1 .

Calibrated data are used to determine the stellar uniform disk angular diameter $\theta_{\mathrm{UD}}$ and limb darkened angular diameter $\theta_{\mathrm{LD}}$ by fitting the functions expressed in Hanbury Brown et al. (1974). An estimate of the star's temperature and gravity based on spectra are used to determine $H$-band limb-darkening coefficients from Claret \& Bloemen (2011). These quantities are iterated upon with the final stellar parameters (see Section 3) to determine the final coefficient used in the limb darkened diameter solution, $\mu_{\mathrm{H}}=0.3512 \quad$ (e.g., see Boyajian et al. 2012, 2013). We measure the angular diameter of 70 Vir to be $\theta_{\mathrm{UD}}=0.967 \pm 0.004$ and $\theta_{\mathrm{LD}}=0.998 \pm 0.005$ milliarcseconds. Figure 1 shows the interferometric data along with the best fit limb-darkened visibility function; the angular diameter measurements may be found in Table 2 .

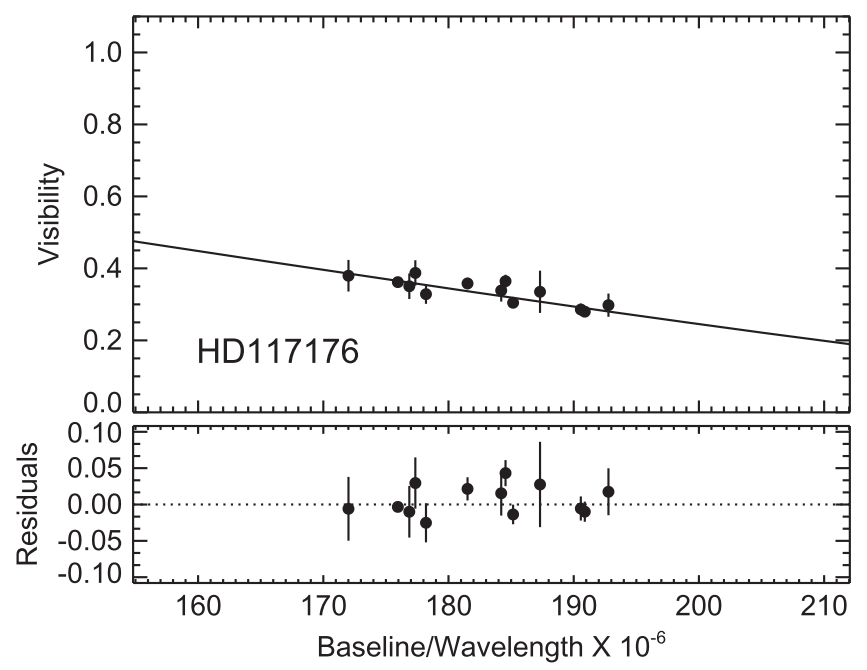

Figure 1. Plot of calibrated interferometric visibilities and the limb-darkened angular diameter fit for 70 Vir. The interferometric observations are described in Section 2.

Table 2

Stellar Properties For 70 Vir

\begin{tabular}{|c|c|c|c|}
\hline Parameter & $\begin{array}{c}\text { Value } \\
\text { Spectroscopic }\end{array}$ & $\begin{array}{c}\text { Value } \\
\text { Interferometric }\end{array}$ & $\begin{array}{c}\text { Section } \\
\text { Reference }\end{array}$ \\
\hline$\theta_{\mathrm{UD}}(\mathrm{mas})$ & $\cdots$ & $0.967 \pm 0.004$ & Section 2 \\
\hline$\theta_{\mathrm{LD}}(\mathrm{mas})$ & $\ldots$ & $0.998 \pm 0.005$ & Section 2 \\
\hline $\begin{array}{l}F_{\text {bol }}\left(10^{-8}\right. \\
\left.\quad \operatorname{erg~s}^{-1} \mathrm{~cm}^{-2}\right)\end{array}$ & $\cdots$ & $28.050 \pm 0.562$ & Section 3.1 \\
\hline Luminosity $\left(L_{\odot}\right)$ & $\cdots$ & $2.827 \pm 0.062$ & Section 3.1 \\
\hline Radius $R_{*}\left(R_{\odot}\right)$ & $1.94 \pm 0.05$ & $1.9425 \pm 0.0272$ & $\begin{array}{l}\text { Section } 3.2 \\
\text { Section } 3.1\end{array}$ \\
\hline$T_{\text {eff }}(\mathrm{K})$ & $5439 \pm 44$ & $5393 \pm 30$ & Section 3.1 \\
\hline$[\mathrm{Fe} / \mathrm{H}]$ & $-0.09 \pm 0.03$ & $\cdots$ & Section 3.2 \\
\hline$v \sin i\left(\mathrm{~km} \mathrm{~s}^{-1}\right)$ & $1.56 \pm 0.50$ & $\cdots$ & Section 3.2 \\
\hline $\log g$ & $3.90 \pm 0.06$ & $\cdots$ & Section 3.2 \\
\hline Mass $M_{*}\left(M_{\odot}\right)$ & $1.09 \pm 0.02$ & $\ldots$ & Section 3.2 \\
\hline Age (Gyr) & $7.77 \pm 0.51$ & $\cdots$ & Section 3.2 \\
\hline
\end{tabular}

Note. For details, see Section 3.

\section{STELLAR PROPERTIES}

\subsection{Interferometry}

The stellar angular diameter measured with interferometry in Section 2 may be used in combination with the trigonometric parallax from Hipparcos to derive the physical linear radius of the star, $R$, using trigonometry. The most direct way to measure the effective temperature of a star is via the Stefan-Boltzmann equation, $L=4 \pi R^{2} \sigma T_{\text {eff }}^{4}$, rearranged to yield

$$
T_{\text {eff }}=2341\left(F_{\mathrm{bol}} / \theta_{\mathrm{LD}}^{2}\right)^{0.25},
$$

where the constant 2341 absorbs the conversion constants assuming using units of $F_{\mathrm{bol}}$ in $10^{-8} \mathrm{erg} \mathrm{s}^{-1} \mathrm{~cm}^{-2}$ and the limbdarkened angular diameter $\theta_{\mathrm{LD}}$ in milliarcseconds (mas).

The bolometric flux is measured by normalizing a G5 V spectral template from the Pickles (1998) library to broadband photometry and spectrophotometry in the literature. Details of this method are described in van Belle et al. (2008) and 


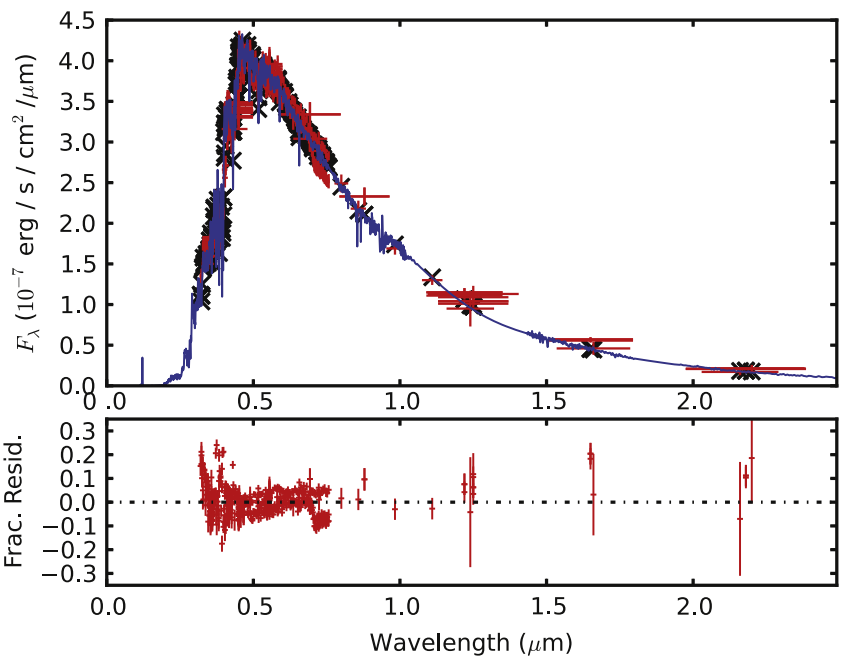

Figure 2. Upper panel: (1) the blue curve is a G5 V spectral template from the Pickles (1998) library, (2) the red crosses are literature photometry and spectrophotometry data of 70 Vir with errors in $y$-direction and filter bandwidths in $x$-direction, (3) the black X-shapes represent the specific flux values of the spectral template at the central wavelength of the filter of the respective literature photometry data point. Lower panel: the red crosses represent the fractional residuals to the fit. The high density of photometry points toward the blue end of the spectrum stems from the three spectrophotometry data sets in the literature for this star. For more details, see Section 3.1 .

von Braun et al. (2014). For 70 Vir, we use photometry from the following references: Johnson \& Morgan (1953), Gutierrez-Moreno et al. (1966), Johnson et al. (1966), Cowley et al. (1967), Pfleiderer et al. (1966), Jerzykiewicz \& Serkowski (1966), Piirola (1976), Johnson \& Harris (1954), Argue (1963), Serkowski (1961), Häggkvist \& Oja (1966), Oja (1985), Mermilliod (1986), Jennens \& Helfer (1975), Moffett \& Barnes (1979), Ducati (2002), Johnson et al. (1968), Beichman et al. (1988), Cutri et al. (2003), Gezari et al. (1999), Oja (1996), Dean (1981), Olsen (1994), Jasevicius et al. (1990), Rufener \& Nicolet (1988), Häggkvist \& Oja (1970), Kornilov et al. (1991), Johnson \& Mitchell (1975), and Smith et al. (2004). We also use the spectrophotometry data from Kharitonov et al. (1988), Glushneva (1998), and Burnashev (1985).

The fit (see Figure 2) produces a bolometric flux $F_{\text {bol }}=(28.050 \pm 0.0248) \times 10^{-8} \mathrm{erg} \mathrm{s}^{-1} \mathrm{~cm}^{-2}$. We note that the quoted uncertainty is statistical only and thus does not account for absolute errors in the templates, uncertain photometric zero-points, or other effects such as the ones outlined in Section 2.2 of von Braun et al. (2014). We follow the arguments in Sections 3.2.1 and 3.2.2 of Bohlin et al. (2014) and add a $2 \%$ error in quadrature to account for a more realistic representation of the true uncertainties (see also the appendix in Bessell \& Murphy 2012). The final $F_{\text {bol }}$ and associated uncertainty values are presented in Table 2, along with the $T_{\text {eff }}$ derived from Equation (1) using $F_{\text {bol }}$ and $\theta_{\mathrm{LD}}$.

\subsection{Spectroscopy}

We model two spectra of 70 Vir, taken on 2009 July 3 with Keck/HIRES. The spectra are modeled using the Spectroscopy Made Easy (SME) package (Valenti \& Piskunov 1996; Valenti $\&$ Fischer 2005). SME employs an iterative mode using results of the model atmosphere analysis in combination with the Yonsei-Yale model isochrones (Demarque et al. 2004) in order to produce self-consistent results with the measured surface gravity (Valenti et al. 2009). The results of the spectroscopic modeling (surface gravity $\log g$, rotational velocity $v \sin i$, atmospheric abundance $[\mathrm{Fe} / \mathrm{H}]$, and effective temperature $T_{\text {eff }}$ ) and stellar isochrone solution (mass $M_{*}$, radius $R_{*}$, and age) are presented in Table 2. The values for effective temperature measured with interferometry and derived with spectroscopic modeling agree well. Likewise, there is excellent agreement with the radius predicted by model isochrones and the directly measured radius with interferometry. The introduction of the interferometric data set not only allows for an empirically based consistency check with the results from stellar atmosphere and evolutionary codes, but also reduces the uncertainties in the stellar parameters beyond the capabilities of current methods employing models $\left(\sigma T_{\text {eff }}\right.$ and $\sigma R_{*}$ are $85 \%$ and $47 \%$ lower, respectively).

\subsection{Stellar Abundances}

There are at least 17 different groups who have measured the stellar abundances in 70 Vir, for example Zhao et al. (2002) and da Silva et al. (2011). Given the close proximity of the host star $(\sim 18 \mathrm{pc})$ and bright $V$ magnitude, elements from Lithium to Europium have been measured within 70 Vir. Per the analysis of Hinkel et al. (2014), the abundance measurements as determined by each group were renormalized to the Lodders et al. (2009) solar abundance scale. The maximum variation between datasets for each element, or the spread, was determined in order to better characterize the consistent measurement of that element abundance. For $70 \mathrm{Vir}$, the median value for $[\mathrm{Fe} / \mathrm{H}]=-0.01 \mathrm{dex}$, while the spread was 0.41 dex, since the renormalized Laird (1985) determination found $[\mathrm{Fe} / \mathrm{H}]=-0.2$ dex and renormalized Valenti \& Fischer (2008) determined $[\mathrm{Fe} / \mathrm{H}]=0.2 \mathrm{dex}$. In the analysis of the Hypatia Catalog (Hinkel et al. 2014), to ensure abundances were copacetic, a star was not considered when the spread of the catalog abundances was greater than the error bar associated with that element, in this case \pm 0.05 dex. Therefore, because of the discrepancy in $[\mathrm{Fe} / \mathrm{H}]$ between datasets, $70 \mathrm{Vir}$ was not incorporated into the analysis of Hypatia.

Despite the $\sim 30$ elements that have been measured in $70 \mathrm{Vir}$, the majority of them suffer from inconsistent measurements between groups. The only elements that do not have a spread greater than the respective error bar are for cases where only one literature source has measured that element, or when the spread is 0.0 dex. In these cases, the renormalized abundances are $[\mathrm{Li} / \mathrm{Fe}]=-1.54 \mathrm{dex}$ and $[\mathrm{Eu} / \mathrm{Fe}]=0.01 \mathrm{dex}($ Gonzalez \& Laws 2007); [K/Fe $]=-0.21$ dex (Zhao et al. 2002); $[\mathrm{ZrII} / \mathrm{Fe}]=$ -0.13 (Mashonkina \& Gehren 2000); [VII/Fe] $=-0.09$ dex and $[\mathrm{CrII} / \mathrm{Fe}]=-0.42 \mathrm{dex}$ (Takeda 2007); and $[\mathrm{Sr} / \mathrm{Fe}]=0.04 \mathrm{dex}$, $[\mathrm{Zr} / \mathrm{Fe}]=0.09 \mathrm{dex},[\mathrm{CeII} / \mathrm{Fe}]=0.08 \mathrm{dex}(\mathrm{da}$ Silva et al. 2011). While many of these abundances are sub-solar, not much can be said given their varying nucleosynthetic origins.

\section{A REFINED PLANETARY ORBIT}

Here we present new RV data for 70 Vir, a revised Keplerian orbital solution for the planet, and an accurate transit ephemeris for $70 \mathrm{Vir}$.

\subsection{Spectra Acquisition}

Previously published data for 70 Vir includes 169 measurements acquired with the Hamilton Echelle Spectro- 
graph (Vogt 1987) on the $3.0 \mathrm{~m}$ Shane Telescope at Lick Observatory (Marcy \& Butler 1996; Butler et al. 2006; Fischer et al. 2014) and 35 measurements acquired with the ELODIE spectrograph (Baranne et al. 1996) on the $1.93 \mathrm{~m}$ telescope at Observatoire de Haute-Provence (Naef et al. 2004). We add to these time series 59 new measurements acquired with the HIRES echelle spectrometer (Vogt et al. 1994) on the $10.0 \mathrm{~m}$ Keck I telescope. H\&K emission measured from the Keck spectra show that $70 \mathrm{Vir}$ is a relatively quiet star. We show the complete dataset of 263 measurements in Table 3, where the fourth column indicates the source of the measurements. These data represent a baseline of $\sim 26$ years of monitoring 70 Vir.

\subsection{Keplerian Orbital Solution}

The revised Keplerian orbital solution to the RV data in Table 3 used RVLIN; a partially linearized, least-squares fitting procedure described in Wright \& Howard (2009). Parameter uncertainties were estimated using the BOOTTRAN bootstrapping routines described in Wang et al. (2012). The resulting orbital solution is shown in Table 4 and in Figure 3.

For each bootstrapping realization, the fit produces offsets for each dataset with respect to the Lick Hamilton dataset. These are fit as two additional free parameters in the Keplerian orbital fit described above. We find the offsets to be 48.4 and $-74.6 \mathrm{~m} \mathrm{~s}^{-1}$ for data from ELODIE and HIRES, respectively. The $\chi_{\text {red }}^{2}$ and rms scatter of the residuals (see Table 4) are consistent with the measurement uncertainties shown in Table 3 . Note that we added a stellar jitter noise component of $3 \mathrm{~m} \mathrm{~s}^{-1}$ in quadrature with the measurement uncertainties (Butler et al. 2006). We find no evidence for a linear RV trend in the fit residuals shown in the right panel of Figure 3.

We performed a further analysis of the data to search for signatures of possible additional planets. Figure 4 shows the Lomb-Scargle periodogram (Horne \& Baliunas 1986; Scargle 1982) of the best-fit residuals, which shows no dominant peak. Since the lower-precision ELODIE and Hamilton data might obscure a low-amplitude signal detectable in the HIRES data, we have also examined the HIRES data alone. Figure 4 shows the periodogram of the residuals of the HIRES data to the best fit shown in Table 4.

The presence of many peaks of a similar amplitude in these periodograms is consistent with there being many, low-mass planets of similar RV semi-amplitude in the data, but also consistent with noise. Since the rms of the residuals is consistent with both expectations and measurements of the uncertainties, there is no reason to expect the former, so we conclude that these data contain no evidence of additional periodic astrophysical signals.

\subsection{Transit Ephemeris Refinement}

The transit mid-point epoch shown in Table 4 was calculated with a Monte-Carlo bootstrap, which propagates the uncertainty in this orbital parameter to the time of the transit. This method produces the most accurate ephemeris since the transit times are calculated as part of the orbital fit. Note that if the planet does not transit then the transit mid-point epoch may be considered the time of inferior conjunction. The predicted transit properties of a system depend sensitively on the stellar radius as well as the planetary parameters. We adopt our interferometric measured radius from Table 2 which has an uncertainty of only $1.5 \%$. The minimum mass of the planet is
Table 3

70 Vir Radial Velocities

\begin{tabular}{lccl}
\hline \hline $\begin{array}{l}\text { Date } \\
(\mathrm{JD}-2,440,000)\end{array}$ & $\begin{array}{c}\mathrm{RV} \\
\left(\mathrm{m} \mathrm{s}^{-1}\right)\end{array}$ & $\begin{array}{c}\sigma \\
\left(\mathrm{m} \mathrm{s}^{-1}\right)\end{array}$ & $\begin{array}{l}\text { Telescope } \\
\text { Instrument }\end{array}$ \\
\hline 7195.02270 & -155 & 13 & Hamilton \\
7195.02790 & -146 & 13 & Hamilton \\
7195.03310 & -146 & 13 & Hamilton \\
7224.84280 & 145 & 10 & Hamilton \\
7224.84810 & 146.0 & 9.6 & Hamilton \\
7224.85300 & 144 & 10 & Hamilton \\
7373.71740 & 109.7 & 9.4 & Hamilton \\
7373.72130 & 115.1 & 9.5 & Hamilton \\
7373.72640 & 114.7 & 8.5 & Hamilton \\
7578.89680 & 232.3 & 9.3 & Hamilton \\
\hline
\end{tabular}

(This table is available in its entirety in machine-readable form.)

Table 4

Keplerian Orbital Model

\begin{tabular}{lc}
\hline \hline Parameter & Value \\
\hline 70 Vir b $P($ days $)$ & $116.6926 \pm 0.0014$ \\
$T_{c}^{\mathrm{a}}(\mathrm{JD}-2,440,000)$ & $16940.258 \pm 0.084$ \\
$T_{p}^{\mathrm{b}}(\mathrm{JD}-2,440,000)$ & $7239.7091 \pm 0.11$ \\
$e$ & $0.399 \pm 0.002$ \\
$\omega\left(\right.$ degree $\left.^{2}\right)$ & $358.8 \pm 0.3$ \\
$K\left(\mathrm{~m} \mathrm{~s}^{-1}\right)$ & $315.7 \pm 0.7$ \\
$M_{p} \sin ^{i}\left(M_{J}\right)$ & $7.40 \pm 0.02$ \\
$a(\mathrm{AU})$ & $0.481 \pm 0.003$ \\
System properties & \\
$\gamma\left(\mathrm{m} \mathrm{s}^{-1}\right)$ & $22.94 \pm 0.59$ \\
Measurements and model $_{N_{\text {obs }}}$ & \\
rms $\left(\mathrm{m} \mathrm{s}^{-1}\right)$ & 263 \\
$\chi_{\text {red }}^{2}$ & 6.08 \\
\hline
\end{tabular}

Notes.

a Time of mid-transit.

$\mathrm{b}$ Time of periastron passage.

larger than a Jupiter mass and we approximate the radius of the planet as $R_{p}=1.0 R_{J}$, based upon the mass-radius relationship described by Kane \& Gelino (2012). These properties, combined with the orbital solution of Table 4 , result in a transit probability of $2.27 \%$, a predicted transit duration of 0.66 days, and a transit depth of $0.3 \%$. The transit mid-point uncertainty shown in Table 4 is 0.084 days, or 121 minutes. Therefore the transit window is dominated by the transit duration rather than the mid-point uncertainty, which is a favorable scenario for photometric follow-up. Our procedure is to use a calculated value for $T_{c}$ as close as possible to the conclusion of observations. However, the baseline of the RV observations described in Section 4.1 is long enough such that there is very little increase in the size of this transit window for the foreseeable future. The uncertainty in the predicted transit time subsequent to that shown in Table 4 has an uncertainty that is less than a minute larger. Kane \& von Braun (2008) have also shown that the transit probability is a strong function of both the eccentricity and the argument of periastron. For 

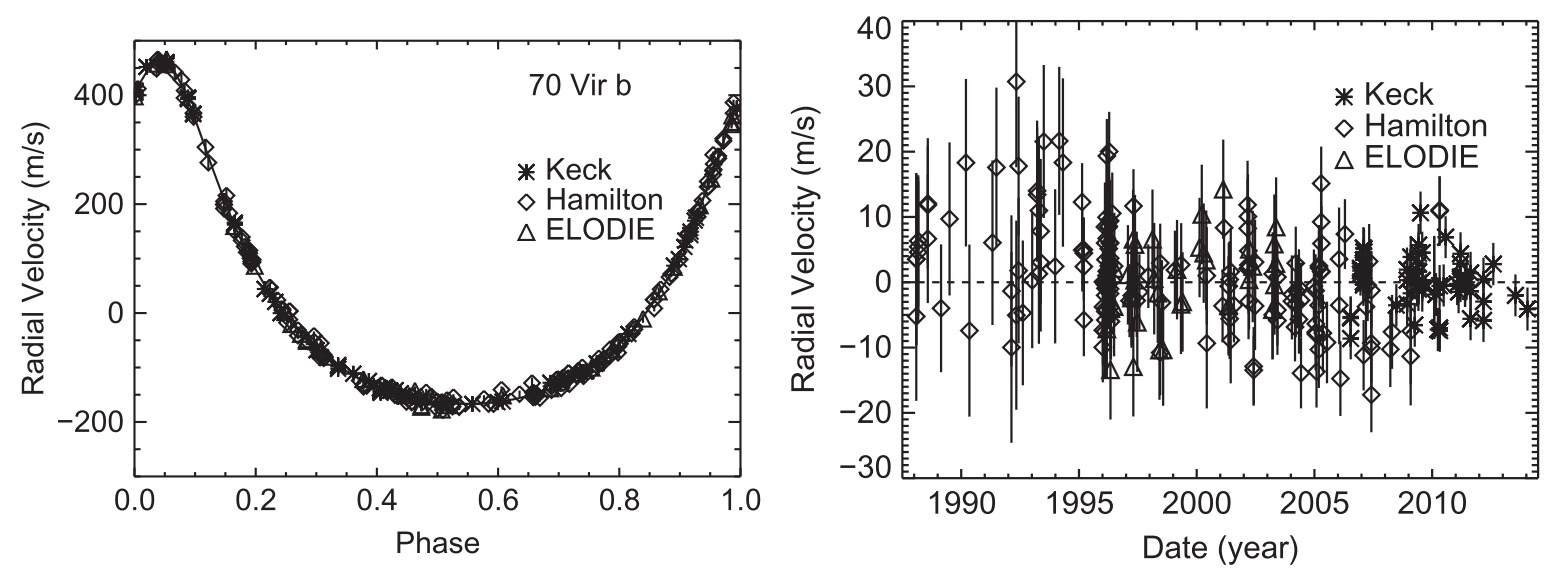

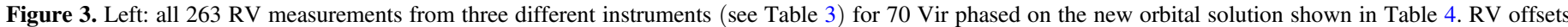
between datasets have been accounted for in this figure. Right: residual velocities with respect to the best orbital solution.
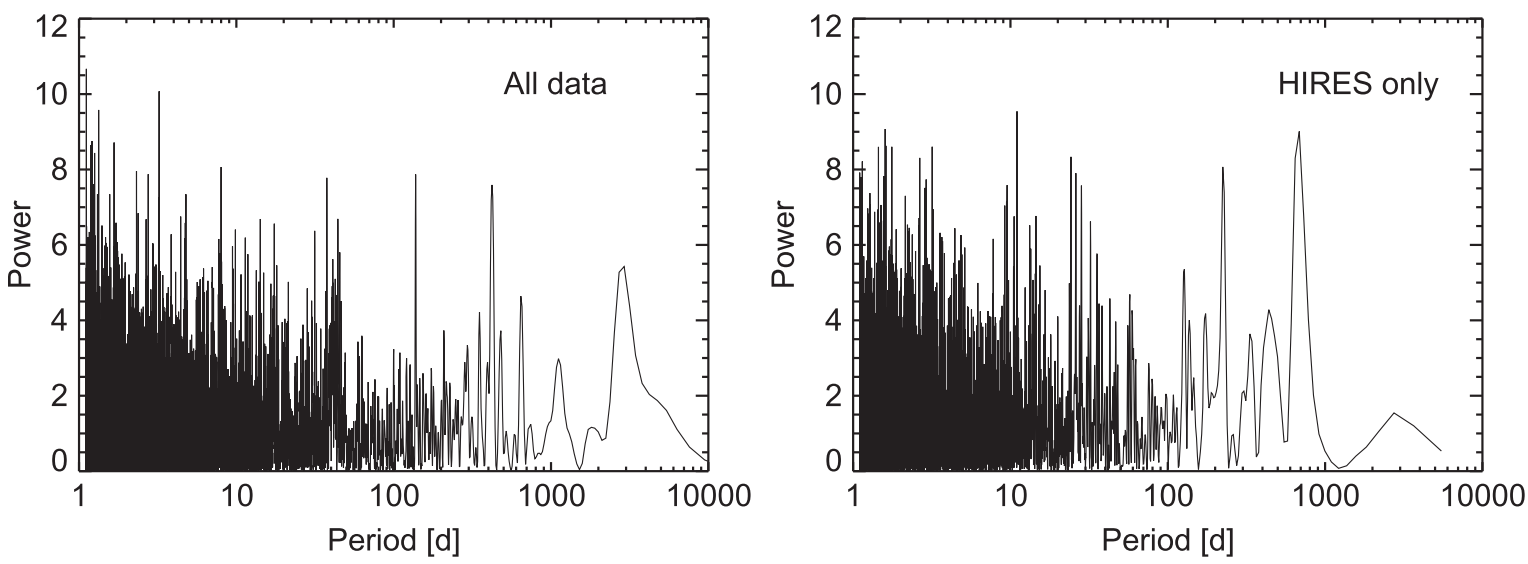

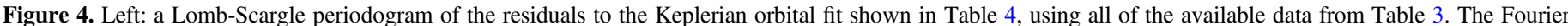

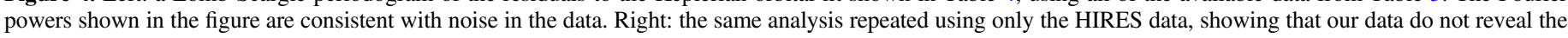
presence of additional planets.

example, if the eccentricity of the planet were zero, the transit probability and duration would be $1.92 \%$ and 0.71 days, respectively. Thus the orientation of the $70 \mathrm{Vir} b$ orbit results in a slightly enhanced transit probability relative to a circular orbit.

\section{SYSTEM HABITABLE ZONE}

The fundamental stellar parameters from Table 2 provide the means to investigate the $\mathrm{HZ}$ of the system and the potential for terrestrial planets in that region. Previous studies of the $70 \mathrm{Vir}$ HZ include those of Jones et al. (2006), who calculated HZ boundaries for a selection of known exoplanetary systems using the estimated ages of stars to determine on-going habitability. Sándor et al. (2007) studied stability regions in the $70 \mathrm{Vir}$ system and concluded that the system is unlikely to host HZ planets. These previous studies used the older HZ boundaries of Kasting et al. (1993). Here we revisit the HZ properties of 70 Vir using the revised system parameters presented here along with the updated HZ calculations of Kopparapu et al. (2013, 2014).

We adopt the definitions of "conservative" and "optimistic" HZ models described by Kane et al. (2013). The conservative HZ use boundaries based upon runaway and maximum greenhouse climate models, whereas the optimistic HZ extends these boundaries based on assumptions regarding the amount of time that Venus and Mars were able to retain liquid water on their surfaces (Kopparapu et al. 2013). The accuracy to which these boundaries can be determined rely on robust determinations of the stellar parameters (Kane 2014) which, in this case, have exceptionally small related uncertainties (see Table 2) such that the HZ boundary uncertainties are negligible. $\mathrm{HZ}$ calculations for all known exoplanetary systems are available using the same methodology through the Habitable Zone Gallery (Kane \& Gelino 2012).

Figure 5 shows a top-down view of the $70 \mathrm{Vir}$ system where the solid line indicates the Keplerian orbit of the planet using the orbital parameters of Table 4. The HZ is depicted by the shaded region where the light gray represents the conservative $\mathrm{HZ}$ and the dark gray is the optimistic extension to the HZ. The conservative $\mathrm{HZ}$ covers the region 1.63-2.92 AU from the host star and the optimistic $\mathrm{HZ}$ increases this region to 1.29-3.08 AU.

Although the confirmed planet is clearly interior to the $\mathrm{HZ}$, we performed stability simulations to investigate whether the relatively large mass of the planet and the eccentricity of its orbit exclude the presence of a hypothetical Earth-mass planet in the HZ. To accomplish this, we performed dynamical simulations using $N$-body integrations with the Mercury Integrator Package (Chambers 1999). We adopted the hybrid 


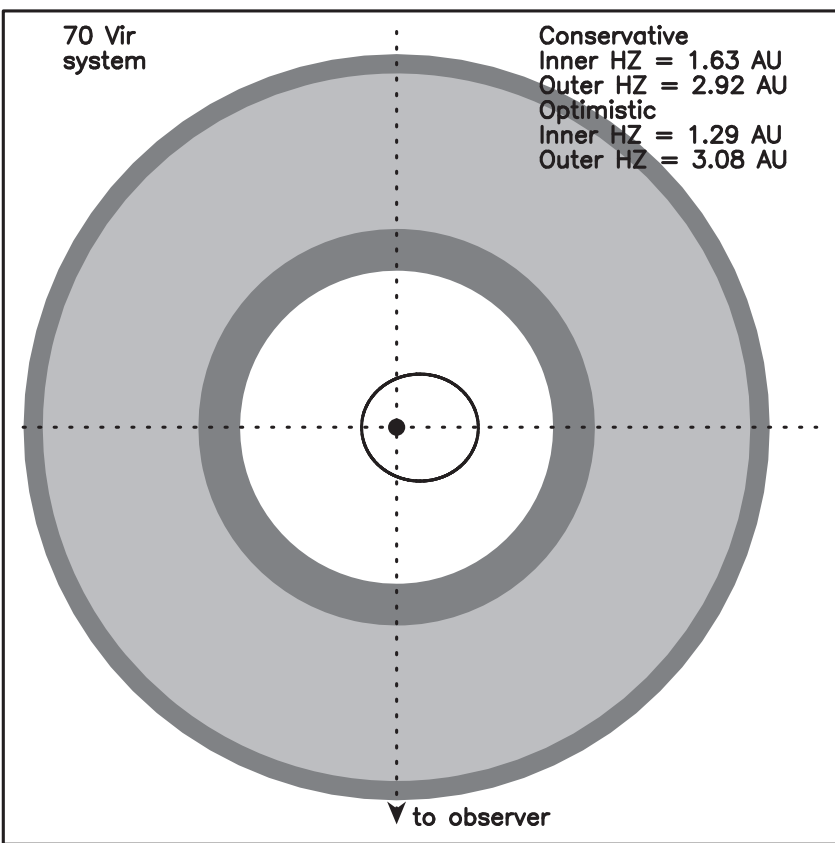

Figure 5. A top-down view of the 70 Vir system showing the extent of the HZ calculated using the stellar parameters of Table 2. The conservative HZ is shown as light-gray and optimistic extension to the HZ is shown as dark-gray. The revised Keplerian orbit of the planet from Table 4 is overlaid.

symplectic/Bulirsch-Stoer integrator and used a Jacobi coordinate system, which provides more accurate results for multiplanet systems (Wisdom \& Holman 1991; Wisdom 2006), except in cases of close encounters (Chambers 1999). We inserted the hypothetical planet in a circular orbit at each of the four optimistic and conservative $\mathrm{HZ}$ boundaries. The integrations were performed for a simulation of $10^{6}$ years, in steps of 100 years, starting at the present epoch.

Assuming that the system is coplanar with an inclination of $90^{\circ}$, our simulations show that the hypothetical systems all remain stable for the full duration of the simulations. The eccentricity of the hypothetical planet oscillates over the course of the simulation with a range of $0.00-0.35,0.04-0.30$, $0.05-0.22$, and $0.03-0.18$ for the optimistic inner, conservative inner, conservative outer, and optimistic outer boundaries, respectively. These eccentricities do not necessarily rule out habitability of the planet, depending on the dynamics of the planetary atmosphere (Williams \& Pollard 2002; Kane \& Gelino 2012c). Note that stability at the HZ boundaries does not guarantee stability within the boundaries, as that is a complex function of orbital distance, phase, and eccentricity.

Since the mass of the inner planet depends on the inclination of the system $\left(M_{p} \sin i=7.40 M_{J}\right)$, we performed simulations that determine the system inclination where the mass of the inner planet causes the orbit of the outer planet to become unstable. These stability threshold inclinations for the four boundaries are $24^{\circ}, 25^{\circ}, 10^{\circ}$, and $3^{\circ}$ for the optimistic inner, conservative inner, conservative outer, and optimistic outer boundaries, respectively. Shown in Figure 6 are the simulation results at the stability threshold inclination for the inner optimistic and conservative HZ boundaries. Each panel shows the eccentricity oscillations for the 50,000 years leading up to the instability event. Even though the inner conservative HZ boundary is farther away from the inner planet, the orbital period at that boundary places the outer planet closer to an orbital resonance with the inner planet than an orbit at the inner optimistic HZ boundary. Thus the planet remains stable for less time at the former than the latter.

\section{PHOTOMETRIC OBSERVATIONS}

We have been monitoring 70 Vir for two decades with the T4 $0.75 \mathrm{~m}$ automatic photoelectric telescope (APT) located at Fairborn Observatory in southern Arizona. The T4 APT observes in the Strömgren $b$ and $y$ pass bands with an EMI 9124QB photomultiplier tube (PMT) as the detector. The automated photometer has a Fabry lens placed behind the focalplane diaphragm that projects a fixed image of the primary mirror (illuminated by the star) onto the photo-cathode of the PMT. Thus, slight motions of the star within the diaphragm during an integration do not translate into image motion on the PMT cathode. The instrumentation and observing strategy result in the data being close to the photon/scintillation noise limit with far less correlated noise than is typical of CCD photometry which suffers from, for example, intra-pixel sensitivity. The data are thus assumed to be uncorrelated in the subsequent analysis. As an additional verification of validity of this assumption, each of the APT integrations are divided into a series of $0.1 \mathrm{~s}$ integrations and saved for quality control and trouble shooting purposes. Histograms of the subinterval data and computed Geneva statistics are used to verify that the data are Gaussian and determine if there are trends, cycles, spikes, or drops in the photon counts during the integration that require further investigation. The T4 APT, its photometer, observing techniques, data reduction procedures, and photometric precision are described in further detail in Henry (1999).

The comparison star HD $117304(\mathrm{C} 1: \quad V=5.65$, $B-V=1.05$, K0 III) has been used for all 23 observing seasons since 1993, while comparison star HD 112503 (C2: $V=6.81, B-V=0.47$, F7 IV) has been used only for the past 19 observing seasons beginning in 1997 because it was chosen to replace a previous comparison star recognized to be a low-amplitude variable after the first four years. T4 has acquired 2051 good differential observations with $\mathrm{C} 1$ over the past 23 years and 1897 good observations with $\mathrm{C} 2$ in the past 19 observing seasons. During the course of our analysis, we recognized that comparison star $\mathrm{C} 1$ exhibited very lowamplitude variability at times; therefore, in this paper, we present the results of our analysis of the 1897 differential magnitudes of $70 \mathrm{Vir}$ with respect to comparison star HD 112503 (C2).

The 1897 differential magnitudes computed with C2 are plotted in the top panel of Figure 7. To increase the precision of the differential magnitudes, we combined the $b$ and $y$ observations into a single $(b+y) / 2$ "pass band." We also normalized each observing season to have the same mean magnitude as the first, thus making our search for short-period variability and shallow transits more sensitive. The nightly normalized observations scatter about their grand mean, indicated by the straight line in the top panel, with a standard deviation $0.00270 \mathrm{mag}$. This is slightly larger than our typical measurement precision given above and may indicate slight residual variability in $70 \mathrm{Vir}$ and/or the comp star HD 112503.

The observations are replotted in the middle panel of Figure 7, where they have been phased with the time of conjunction and the orbital period from Table 4. A leastsquares sine fit on the 116.6926 day radial velocity period gives 

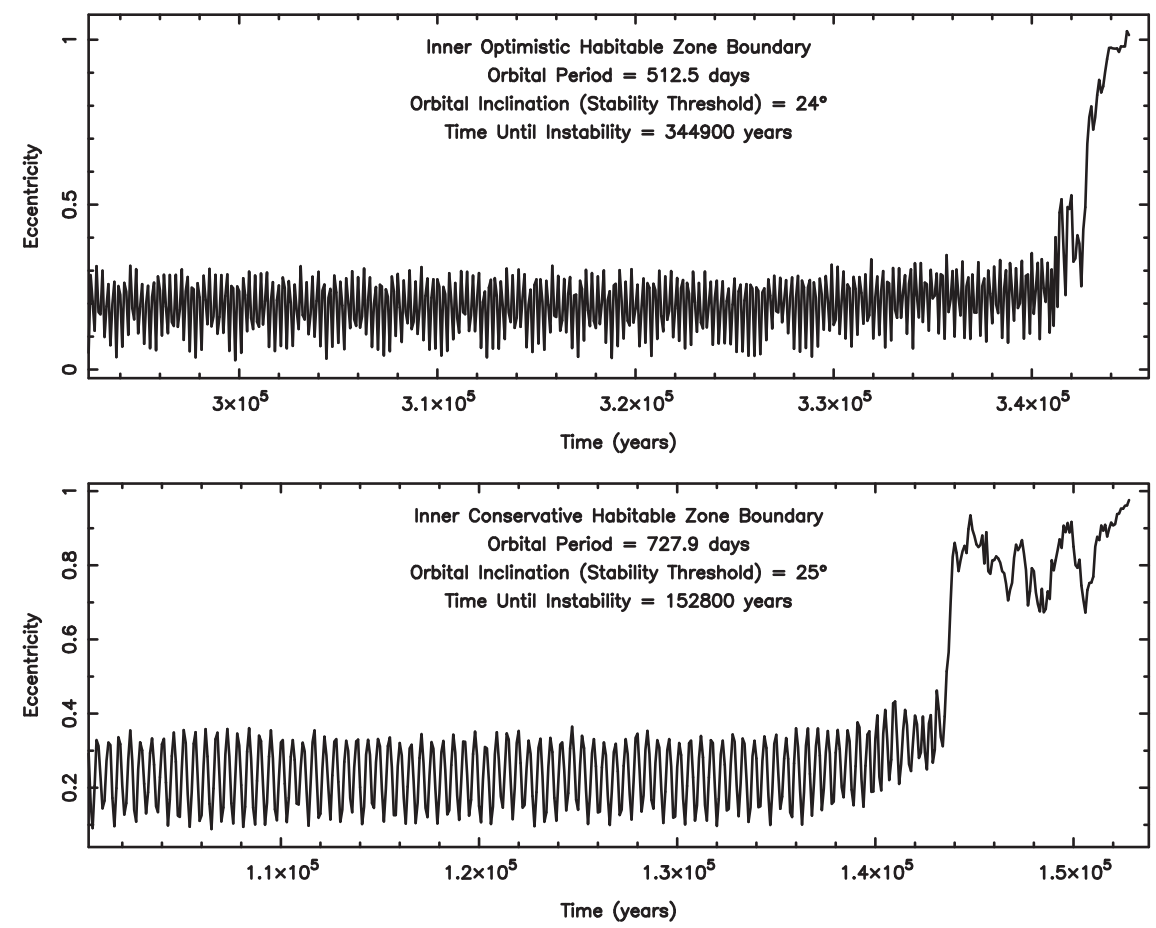

Figure 6. Stability simulations for a hypothetical Earth-mass planet in the $\mathrm{HZ}$ of the 70 Vir system. Each panel shows the eccentricity oscillations for the 50,000 years leading up to the ejection of the outer planet. Top panel: the Earth-mass planet remains stable at the inner boundary of the optimistic HZ for system inclinations $>24^{\circ}$, otherwise the inner planet causes the outer planet to be ejected after $\sim 350,000$ years. Bottom panel: the Earth-mass planet remains stable at the inner boundary of the conservative $\mathrm{HZ}$ for system inclinations $>25^{\circ}$, otherwise the inner planet causes the outer planet to be ejected after $\sim 150,000$ years.

a formal semi-amplitude of just $0.000352 \pm 0.000076 \mathrm{mag}$, thus confirming that the periodic radial velocity variations are due to planetary reflex motion and not to intrinsic stellar brightness variations (see, e.g., Queloz et al. 2001; Paulson et al. 2004; Boisse et al. 2012).

The observations within \pm 0.03 phase units of the predicted transit time are plotted in the bottom panel of Figure 7 . The solid curve shows the predicted transit phase $(0.0)$, the transit depth $(0.3 \%$ or $0.00325 \mathrm{mag})$, and transit duration $( \pm 0.003$ phase units) computed as described in Section 4.3 above. The horizontal line below the transit window represents the transit mid-point uncertainty. While the second half of the transit window is not well covered by our observations, there are a total of 27 observations within the transit window that have a mean of $-1.773035 \pm 0.000416$ mag and 1870 observations that fall outside the transit window and have a mean of $-1.772784 \pm 0.000063 \mathrm{mag}$. Thus, our "observed" transit depth is $-0.000251 \pm 0.000421 \mathrm{mag}$, which is consistent with zero to three decimal places. Therefore, a central transit of the expected depth and duration occurring at the expected time can be ruled out at the $5 \sigma$ level. Although the data sampling is sufficient to also constrain the absence of transits for almost all impact parameters, the number of data points within the corresponding transit durations will be less, thereby lessening the significance of such constraints. For example, the largest gap in the photometry during the transit window corresponds to $\sim 0.3$ of the central transit duration. This means the impact parameter would need to be $\geqslant 0.95$ to have been completely missed by our data. Ruling out such a range of impact parameters would reduce the posterior transit probability from $2.27 \%$ to $0.11 \%$.

70 Vir is a magnetically inactive star with $\log R_{H K}^{\prime}$ values of -4.99 and -5.116 according to Wright et al. (2004) and
Isaacson \& Fischer (2010), respectively. Wright et al. (2004) give an estimated rotation period of 32 days for $70 \mathrm{Vir}$, based on the star's activity level. However, no reliable rotation period for 70 Vir has been directly measured via rotational modulation of dark starspots or bright $\mathrm{Ca}$ II $\mathrm{H}$ and $\mathrm{K}$ regions across the face of the star (e.g., Henry et al. 1997, 2000; Simpson et al. 2010). We performed periodogram analyses of each individual observing season and of our data set as a whole, and, while we found suggestions of low-amplitude variability, we could not identify any significant period that might be interpreted as the stellar rotation period.

Finally, we look for long-term variability in 70 Vir. Unfortunately, comparison star $\mathrm{C} 1$ has significant long-term variability of several mmag. The yearly mean differential magnitudes (70 Vir-C1) vary over a range $0.0076 \mathrm{mag}$ and have a standard deviation of $0.0023 \mathrm{mag}$ with respect to the grand mean. However, the yearly means of $(70 \mathrm{Vir}-\mathrm{C} 2)$ have both a smaller range and a smaller standard deviation, $0.0041 \mathrm{mag}$ and $0.0012 \mathrm{mag}$, respectively (see Figure 8). Without another good comparison star, we cannot determine whether the variability we see in Figure 8 originates in 70 Vir, the comparison star, or a combination of both. Thus, we can only state that 70 Vir's long-term variability has a range less than $\sim 0.004$ mag.

\section{CONCLUSIONS}

In an era where new planets are being regularly discovered via the transit method, the bright exoplanet host stars still largely belong to those planets that were discovered using the radial velocity method. These are systems that thus provide the greatest access to follow-up investigations due to the relatively large signal-to-noise possibilities presented. Here we have presented new results for the 70 Vir system that includes 

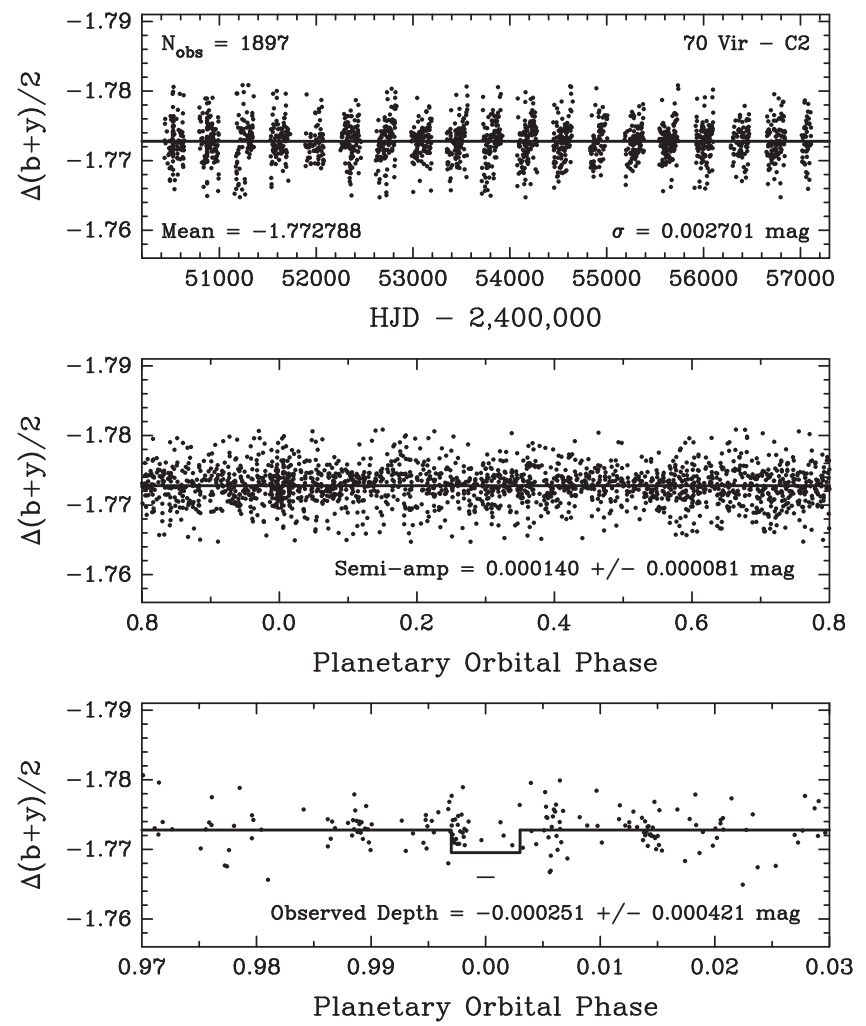

Figure 7. Top: the 1997-2015 normalized differential observations of 70 Vir with respect to comparison star C2, acquired with the T4 $0.75 \mathrm{~m}$ APT between 1997 and 2015. Middle: the observations phased with the 116.6926 day orbital period. The semi-amplitude of a least-squares sine fit to the phase observations is $0.000140 \pm 0.000081 \mathrm{mag}$, consistent with the absense of light variability on the radial velocity period and confirming planetary reflex motion of the star as the cause of the radial velocity variations. Bottom: the observations within \pm 0.03 phase units of the predicted transit time. The solid curve shows the predicted transit time at phase 0.0 , transit depth $(0.3 \%)$ and duration $( \pm 0.003$ phase units) for a central transit of planet $b$. The short horizontal line segment represents the uncertainty in the time of tansit. Our photometry shows that central transits of the expected depth and duration likely do not occur.

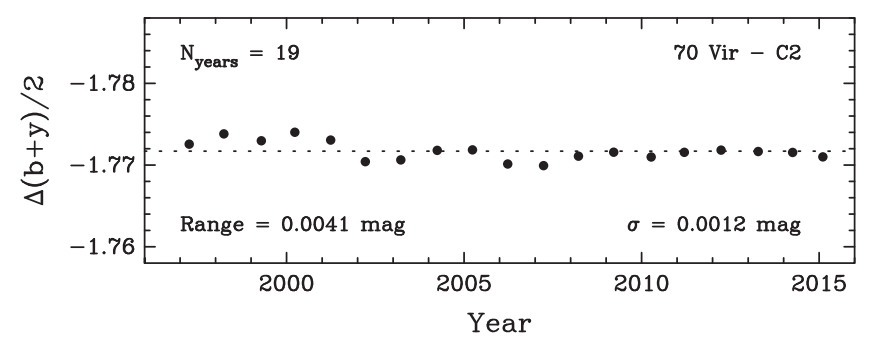

Figure 8. The 1997-2015 yearly mean differential magnitudes 70 Vir minus comparison star $\mathrm{C} 2$. The error bars on the individual seasonal means are slightly smaller than the plotted points. The 19 differential magnitudes vary over a total range of $0.0041 \mathrm{mag}$ and have a standard deviation of $0.0012 \mathrm{mag}$ with respect to the grand mean, indicated by the horizonal dotted line. Without another good comparison star, we are unable to determine whether the variability is intrinsic to $70 \mathrm{Vir}$ or the comparison star, so these values can only be quoted as upper limits of long-term variability in 70 Vir.

detailed characterization of the host star. Our direct measurements of the stellar radius show that, although slightly cooler, $70 \mathrm{Vir}$ is almost twice the size of the Sun. This is consistent with the star being older and more evolved than the Sun. Our new radial velocity data provide an improved Keplerian orbital solution for the planet and further evidence that there are unlikely to be further giants planets within the system. A terrestrial-mass planet may yet exist beneath our detection threshold and so, given the vastly improved stellar properties, we calculated the $\mathrm{HZ}$ boundaries and performed stability simulations within that region. Our simulations show that a terrestrial planet could remain in a stable orbit near the $\mathrm{HZ}$ inner edge for system inclinations $>25^{\circ}$ and close to the outer $\mathrm{HZ}$ edge for almost all system inclinations. Finally, our 19 years of APT photometry confirm that the star is quite stable over long time periods and there is no evidence that the b planet transits the host star (a "dispositive null," as described by Wang et al. 2012), the timing of which we were able to accurately predict from our revised Keplerian orbit. The TERMS compilation of data for this system presented here means that it is now one of the better characterized systems in terms of stellar and planetary parameters.

We thank the anonymous referee for helpful comments which improved the manuscript. S.R.K and N.R.H. acknowledge financial support from the National Science Foundation through grant AST-1109662. T.S.B acknowledges support provided through NASA grant ADAP12-0172. The CHARA Array is funded by the National Science Foundation through NSF grants AST-0606958 and AST-0908253 and by Georgia State University through the College of Arts and Sciences, as well as the W.M. Keck Foundation. G.W.H. acknowledges support from NASA, NSF, Tennessee State University, and the State of Tennessee through its Centers of Excellence program. Y.K.F. and J.T.W. acknowledge support from NASA Keck PI Data Awards, administered by the NASA Exoplanet Science Institute, including awards 2007B N095Hr, 2010A N147Hr, 2011A\&B N141Hr, \& 2012A N129Hr; NASA Origins of Solar Systems grant NNX09AB35G; NASA Astrobiology Institute grant NNA09DA76A; and the Center for Exoplanets and Habitable Worlds (which is supported by the Pennsylvania State University, the Eberly College of Science, and the Pennsylvania Space Grant Consortium). This research has made use of the Habitable Zone Gallery at hzgallery.org. This research has also made use of the SIMBAD and VIZIER Astronomical Databases, operated at CDS, Strasbourg, France (http://cdsweb.u-strasbg.fr/), and of NASA's Astrophysics Data System, of the Jean-Marie Mariotti Center SearchCal service (http://www.jmmc.fr/searchcal), co-developed by FIZEAU and LAOG/IPAG.

\section{REFERENCES}

Argue, A. N. 1963, MNRAS, 125, 557

Baranne, A., Queloz, D., Mayor, M., et al. 1996, A\&AS, 119, 373 Beichman, C. A., Neugebauer, G., Habing, H. J., Clegg, P. E., \& Chester, T. J. 1988, Infrared Astronomical Satellite (IRAS) Catalogs and Atlases, 1 Bessell, M., \& Murphy, S. 2012, PASP, 124, 140 Bohlin, R. C., Gordon, K. D., \& Tremblay, P.-E. 2014, PASP, 126, 711 Boisse, I., Bonfils, X., \& Santos, N. C. 2012, A\&A, 545, 109 Bonneau, D., Clausse, J.-M., Delfosse, X., et al. 2006, A\&A, 456, 789 Bonneau, D., Delfosse, X., Mourard, D., et al. 2011, A\&A, 535, A53 Boyajian, T. S., von Braun, K., van Belle, G., et al. 2012, ApJ, 757, 112 Boyajian, T. S., von Braun, K., van Belle, G., et al. 2013, ApJ, 771, 40 Burnashev, B. I. 1985, BCrAO, 66, 152

Butler, R. P., Wright, J. T., Marcy, G. W., et al. 2006, ApJ, 646, 505

Chambers, J. E. 1999, MNRAS, 304, 793

Claret, A., \& Bloemen, S. 2011, A\&A, 529, A75

Cowley, A. P., Hiltner, W. A., \& Witt, A. N. 1967, AJ, 72, 1334

Cutri, R. M., Skrutskie, M. F., van Dyk, S., et al. 2003, The IRSA 2MASS AllSky Point Source Catalog(NASA/IPAC Infrared Science Archive) da Silva, R., Milone, A. C., \& Reddy, B. E. 2011, A\&A, 526, 71 
Dean, J. F. 1981, SAAOC, 6, 10

Demarque, P., Woo, J.-H., Kim, Y.-C., \& Yi, S. K. 2004, ApJS, 155, 667

Dragomir, D., Kane, S. R., Henry, G. W., et al. 2012, ApJ, 754, 37

Dressing, C. D., \& Charbonneau, D. 2013, ApJ, 767, 95

Ducati, J. R. 2002, yCat, 2237, 0

Fischer, D. A., Marcy, G. W., \& Spronck, J. F. P. 2014, ApJS, 210, 5

Gezari, D. Y., Pitts, P. S., \& Schmitz, M. 1999, yCat, 2225, 0

Glushneva, I. N., Doroshenko, V. T., Fetisova, T. S., et al. 1998, yCat, 3207, 0

Gonzalez, G., \& Laws, C. 2007, MNRAS, 378, 1141

Gutierrez-Moreno, A., et al. 1966, PDAUC, 1, 1

Häggkvist, L., \& Oja, T. 1966, ArA, 4, 137

Häggkvist, L., \& Oja, T. 1970, A\&AS, 1, 199

Hanbury Brown, R., Davis, J., \& Allen, L. R. 1974, MNRAS, 167, 121

Henry, G. W. 1999, PASP, 111, 845

Henry, G. W., Baliunas, S. L., Donahue, R. A., Fekel, F. C., \& Soon, W. H. 2000, ApJ, 531, 415

Henry, G. W., Baliunas, S. L., Donahue, R. A., Soon, W. H., \& Saar, S. H. 1997, ApJ, 474, 503

Henry, G. W., Kane, S. R., Wang, S. X., et al. 2013, ApJ, 768, 155

Hinkel, N. R., Timmes, F. X., Young, P. A., Pagano, M. D., \& Turnbull, M. C. 2014, AJ, 148, 54

Horne, J. H., \& Baliunas, S. L. 1986, ApJ, 302, 757

Huber, D., Silva Aguirre, V., Matthews, J. M., et al. 2014, ApJS, 211, 2

Isaacson, H., \& Fischer, D. 2010, ApJ, 725, 875

Jasevicius, V., Kuriliene, G., Strazdaite, V., et al. 1990, VilOB, 85, 50

Jennens, P. A., \& Helfer, H. L. 1975, MNRAS, 172, 667

Jerzykiewicz, M., \& Serkowski, K. 1966, PASP, 78, 546

Johnson, H. L., \& Harris, D. L. 1954, ApJ, 120, 196

Johnson, H. L., MacArthur, J. W., \& Mitchell, R. I. 1968, ApJ, 152, 465

Johnson, H. L., \& Mitchell, R. I. 1975, RMxAA, 1, 299

Johnson, H. L., Mitchell, R. I., Iriarte, B., \& Wisniewski, W. Z. 1966, CoLPL, 4,99

Johnson, H. L., \& Morgan, W. W. 1953, ApJ, 117, 313

Jones, B. W., Sleep, P. N., \& Underwood, D. R. 2006, ApJ, 649, 1010

Kane, S. R. 2014, ApJ, 782, 111

Kane, S. R., Barclay, T., \& Gelino, D. M. 2013, ApJL, 770, L20

Kane, S. R., \& Gelino, D. M. 2012, PASP, 124, 323

Kane, S. R., \& Gelino, D. M. 2012c, AsBio, 12, 940

Kane, S. R., Howell, S. B., Horch, E. P., et al. 2014, ApJ, 785, 93

Kane, S. R., Mahadevan, S., von Braun, K., Laughlin, G., \& Ciardi, D. R. 2009, PASP, 121, 1386

Kane, S. R., \& von Braun, K. 2008, ApJ, 689, 492

Kasting, J. F., Whitmire, D. P., \& Reynolds, R. T. 1993, Icar, 101, 108

Kharitonov, A. V., Tereshchenko, V. M., \& Knyazeva, L. N. 1988, The Spectrophotometric Catalogue of Stars. Book of reference, ed. A. V. Kharitonov, V. M. Tereshchenko, L. N. Knyazeva, \& Alma-Ata. Nauka (USSR), 478

Kopparapu, R. K. 2013, ApJL, 767, L8

Kopparapu, R. K., Ramirez, R., Kasting, J. F., et al. 2013, ApJ, 765, 131

Kopparapu, R. K., Ramirez, R. M., SchottelKotte, J., et al. 2014, ApJL, 787, L29

Kornilov, V. G., Volkov, I. M., Zakharov, A. I., et al. 1991, TrSht, 63, 4
Laird, J. B. 1985, ApJ, 289, 556

Lodders, K., Palme, H., \& Gail, H.-P. 2009, Landolt-Börnstein-Group VI Astronomy and Astrophysics Numerical Data and Functional Relationships in Science and Technology, Vol. 4B, ed. J. E. Trümper, 44

Marcy, G. W., \& Butler, R. P. 1996, ApJL, 464, L147

Mashonkina, L., \& Gehren, T. 2000, A\&A, 364, 249

Mermilliod, J.-C. 1986, Catalogue of Eggen's UBV data

Moffett, T. J., \& Barnes, T. G., III 1979, PASP, 91, 180

Naef, D., Mayor, M., Beuzit, J. L., et al. 2004, A\&A, 414, 351

Oja, T. 1985, A\&AS, 61, 331

Oja, T. 1996, BaltA, 5, 103

Olsen, E. H. 1994, A\&AS, 106, 257

Paulson, D. B., Saar, S. H., Cochran, W. D., \& Henry, G. W. 2004, AJ, 127,1644

Perryman, M. A. C., Lindegren, L., Arenou, F., et al. 1996, A\&A, 310, L21

Petigura, E. A., Howard, A. W., \& Marcy, G. W. 2013, PNAS, 110, 19273

Pfleiderer, J., Dachs, J., \& Haug, U. 1966, ZA, 64, 116

Pickles, A. J. 1998, PASP, 110, 863

Piirola, V. 1976, HelR, 1, 0

Queloz, D., Henry, G. W., Sivan, J. P., et al. 2001, A\&A, 379, 279

Rufener, F., \& Nicolet, B. 1988, A\&A, 206, 357

Sándor, Zs., Süli, Á, Érdi, B., Pilat-Lohinger, E., \& Dvorak, R. 2007, MNRAS, 375,1495

Scargle, J. D. 1982, ApJ, 263, 835

Serkowski, K. 1961, LowOB, 5, 157

Simpson, E. K., Baliunas, S. L., Henry, G. W., \& Watson, C. A. 2010, MNRAS, 408, 1666

Smith, B. J., Price, S. D., \& Baker, R. I. 2004, ApJS, 154, 673

Takeda, Y. 2007, PASJ, 59, 335

ten Brummelaar, T. A., McAlister, H. A., Ridgway, S. T., et al. 2005, ApJ, 628,453

Valenti, J. A., \& Fischer, D. A. 2005, ApJS, 159, 141

Valenti, J. A., \& Fischer, D. A. 2008, PhST, 130, 4003

Valenti, J. A., Fischer, D., Marcy, G. W., et al. 2009, ApJ, 702, 989

Valenti, J. A., \& Piskunov, N. 1996, A\&AS, 118, 595

van Belle, G. T., \& van Belle, G. 2005, PASP, 117, 1263

van Belle, G. T., van Belle, G., Creech-Eakman, M. J., et al. 2008, ApJS, 176,276

van Leeuwen, F. 2007, A\&A, 474, 653

Vogt, S. S. 1987, PASP, 99, 1214

Vogt, S. S., Allen, S. L., Bigelow, B. C., et al. 1994, in Proc. SPIE, 2198, 362

von Braun, K., Boyajian, T. S., van Belle, G. T., et al. 2014, MNRAS, 438,2413

Wang, S. S., Wright, J. T., Cochran, W., et al. 2012, ApJ, 761, 46

Williams, D. M., \& Pollard, D. 2002, IJAsB, 1, 61

Wisdom, J. 2006, AJ, 131, 2294

Wisdom, J., \& Holman, M. 1991, AJ, 102, 1528

Wright, J. T., \& Howard, A. W. 2009, ApJS, 182, 205

Wright, J. T., Marcy, G. W., Butler, R. P., \& Vogt, S. S. 2004, ApJS, 152,261

Zhao, G., Chen, Y.-Q., \& Qin, H.-M. 2002, ChJAA, 2, 377 